\title{
Laparoscopic right hepatectomy after portal vein embolization in hepatocellular carcinoma
}

\author{
Jaryung HAN, Young Seok HAN* \\ Division of Hepatobiliary Pancreas Surgery and Liver Transplantation, Department of Surgery, \\ Kyungpook National University Hospital, Daegu, Korea
}

Introduction: Portal vein embolization (PVE) has been documented as an essential strategy for patient with small future liver remnant, to minimize postoperative morbidity and mortality. The majority of hepatectomy requiring preoperative PVE is approached using conventional operation because of the anticipated complexity of the case. Laparoscopic liver resection (LLR) has been gradually increased and similar outcomes have been reported when compared to open hepatectomy. However, it is very difficult to find the reports about LLR after PVE. Hence, we will present our experiences and outcomes for LLR after PVE in hepatocellular carcinoma (HCC) patients with significant technical tips.

Methods: We performed laparoscopic right hepatectomy after PVE in 8 HCC patients from 2016 to 2020. The operation was performed within 3 weeks after PVE. We confirmed the atrophy of resected liver and compensatory hypertrophy of future liver remnant using preoperative computed tomography scan. During surgery, individual inflow control was easier because right portal vein had been already occluded.

Results: There was no blood transfusion and open conversion. There was no statistical difference in operation time, intraoperative complications and postoperative morbidity including hospital stay, compared to open hepatectomy.

Conclusions: PVE is very useful procedure even in laparoscopic right hepatectomy as in open hepatectomy. However, caution is needed when PVE is applied cirrhotic livers. Therefore, adequate candidate selection for PVE and technical refinement are needed to decrease morbidity and increase surgical outcomes. 